\title{
THE SAGITAL BALANCE IN IDIOPATIC AND NEUROMUSCULAR SCOLIOSIS
}

\author{
Paulo Alvim Borges ${ }^{1}$, Guilherme Pereira Ocampos ${ }^{1}$, José Antonio Mancuso Filho ${ }^{1}$, Olavo Biraghi Letalf ${ }^{1}$, \\ Raphael Martus Marcon ${ }^{1}$, alexandre Fogaça Cristante $^{1}$
}

\begin{abstract}
Objectives: To describe and compare the distribution of spinopelvic parameters (SPP) in a Brazilian population with idiopathic scoliosis (IS) and neuromuscular scoliosis (NMS), and evaluate the association between pelvic incidence (PI) and lumbar lordosis (LL). Method: Medical records investigation was performed. Sagital balance angles were measured in patients with neuromuscular and idiopathic scoliosis. Results: IS sample means (in degrees): PI 55.55; Sacral Slope (SS) 45.35; Pelvic Tilt (PT) 10.19; Lumbar Lordosis (LL) 43.48; and Thoracic Kyphosis (TK) 32.10. In NMS: PI 53.77; SS 42.31; PT 11.46; LL 49.46; and TK 45.69. No statistically
\end{abstract}

significant differences in PEP distribution were found between the two types of scoliosis $(p=0,057)$. The association between pelvic incidence and lumbar lordosis is low among idiopathic scoliosis $(R=0,074)$ and neuromuscular scoliosis $(R=0,274)$. Conclusion: PEP measurements in a Brazilian population of idiopathic scoliosis and neuromuscular scoliosis patients are similar to those in the international literature and do not differ statistically between them. The association between LL and PI could not be assessed in this study. Level of Evidence IV, Case Series.

Keywords: Scoliosis. Postural balance. Spine. Pelvis.

Citation: Borges PA, Ocampos GP, Mancuso Filho JA, Letaif OB, Marcon RM, Cristante AF. The sagital balance in idiopatic and neuromuscular scoliosis. Acta Ortop Bras. [online] 2014;22(4):179-82. Available from URL: http://www.scielo.br/aob.

\section{INTRODUCTION}

The sagittal balance of the spine has been a topic of discussion in recent years and its importance in the treatment of pathologies of the spine has been increasingly considered..$^{1,2}$

Among the most commonly used sagittal parameters, thoracic kyphosis and lumbar lordosis are already widely known in pediatrics. ${ }^{3,4}$ However, more recently, spinopelvic parameters have gained attention and research of its variation in diverse populations has been studied. ${ }^{5}$

Proper spinopelvic alignment minimizes energy expenditure in moving and keep the horizon look stable, ${ }^{1}$ besides being associated with better quality of life in patients with vertebral deformity. ${ }^{6,7}$ It consists of three parameters: pelvic incidence, Pelvic Tilt and Sacral Tilt.

Although these parameters have been well described in the normal pediatric population, their distribution and relevance in pathologies of the spine are not yet understood, since there are only few publications such as Li et al. ${ }^{2}$ who studied the distribution of spinopelvic angle in patients with adolescent idiopathic scoliosis comparing them with the normal pediatric population. The correlation between some spinopelvic parameters and lumbar lordosis has been described in patients with idiopathic scoliosis, ${ }^{8}$ but has not yet been reported in patients with neuromuscular scoliosis.

This study aims to describe the distribution of spinopelvic parameters in a Brazilian population of patients with idiopathic and neuromuscular scoliosis and to compare them. We will further evaluate the association between pelvic incidence and lumbar lordosis in these populations.

\section{MATERIALS AND METHODS}

The study consisted in the analysis of patients' records with scoliosis treated at the Institute of Orthopedics and Traumatology, Hospital das Clínicas, Universidade de São Paulo (IOT-HCFMUSP) from January 2009 to January 2013, chosen by continuous convenience.

This study was submitted to the institution Research Ethics Committee and approved before its start.

Patients who did not present idiopathic or neuromuscular scoliosis and those who had inadequate radiographic record were excluded from the study.

It was considered as appropriate record panoramic radiographs in profile incidence of the spine that included the limits of $\mathrm{C7}$ vertebra and the femoral head.

All the authors declare that there is no potential conflict of interest referring to this article.

\footnotetext{
1. Institute of Orthopedics and Traumatology, Hospital das Clínicas da Faculdade de Medicina da Universidade de São Paulo, São Paulo,SP, Brazil.

Work developed at Lim 41 - Laboratory of Medical Investigation of the Musculoskeletal System, Department of Orthopedic and Traumatology, Faculdade de Medicina da Universidade de São Paulo, São Paulo, SP, Brazil.

Correspondence: Olavo Biraghi Letaif, Centro de Atendimento ao Traumatizado Raquimedular (CENATRA), Instituto de Ortopedia e Traumatologia da Faculdade de Medicina da Correspondence: Olavo Biraghi Letaif, Centro de Atendimento ao Traumatizado Raquimedular (CENATRA), Instituto de
Universidade de São Paulo. Rua Dr. Ovídio Pires de Campo, 333, 05403-010, São Paulo, SP, Brazil. oletaif@yahoo.com.br
} 
The chart analysis consisted of measuring the spinopelvic ${ }^{1,8}$ and kyphosis and lordosis parameters by Cobb's method, ${ }^{9}$ as follows: (Figure 1)

1. Pelvic Incidence (PI): measure of the angle formed by lines drawn from the midpoint between the center of the femoral heads and the center point of the upper plateau of S1 and a perpendicular to the upper plateau of S1 starting at its center point;

2. Sacral slope (SS): measure of the angle formed by the horizontal line and the line drawn tangential to the upper plateau of $\mathrm{S} 1$;

3. Pelvic Tilt (PT): measure of the angle formed by lines drawn from the midpoint between the centers of the femoral heads and the center point of the upper plateau of S1 and the vertical plumb line to that point;

4. Lumbar lordosis (LL): measure of the angle formed by the tangent lines to the lower plateau of $L 5$ and upper of $L 1$;

5. Thoracic kyphosis (TK): measure of the angle formed by the tangent lines to the lower plateau of T12 and upper of T2.

The first three angles described are said spinopelvic parameters, pelvic incidence being a constant measure intrinsic of each individual, which is calculated by the sum of sacral and pelvic inclinations, according to formula $(\mathrm{Pl}=\mathrm{SS}+\mathrm{PT}){ }^{1,8}$ All measures were calculated in degrees.

The measurements were all performed evenly by digital preoperative radiographs with digital display system-Philips iSite ${ }^{(\mathrm{TM})}$ following a method that has been already validated for measurement by digital radiographs in scoliosis. ${ }^{10}$

For data analysis there were calculated: average and median distribution measurements discriminating populations into idiopathic and neuromuscular and determined their normality by Kolgomorov-Smirnov test, as well as analysis of distribution histograms. These were compared in order to verify whether there are differences and their statistical significance.

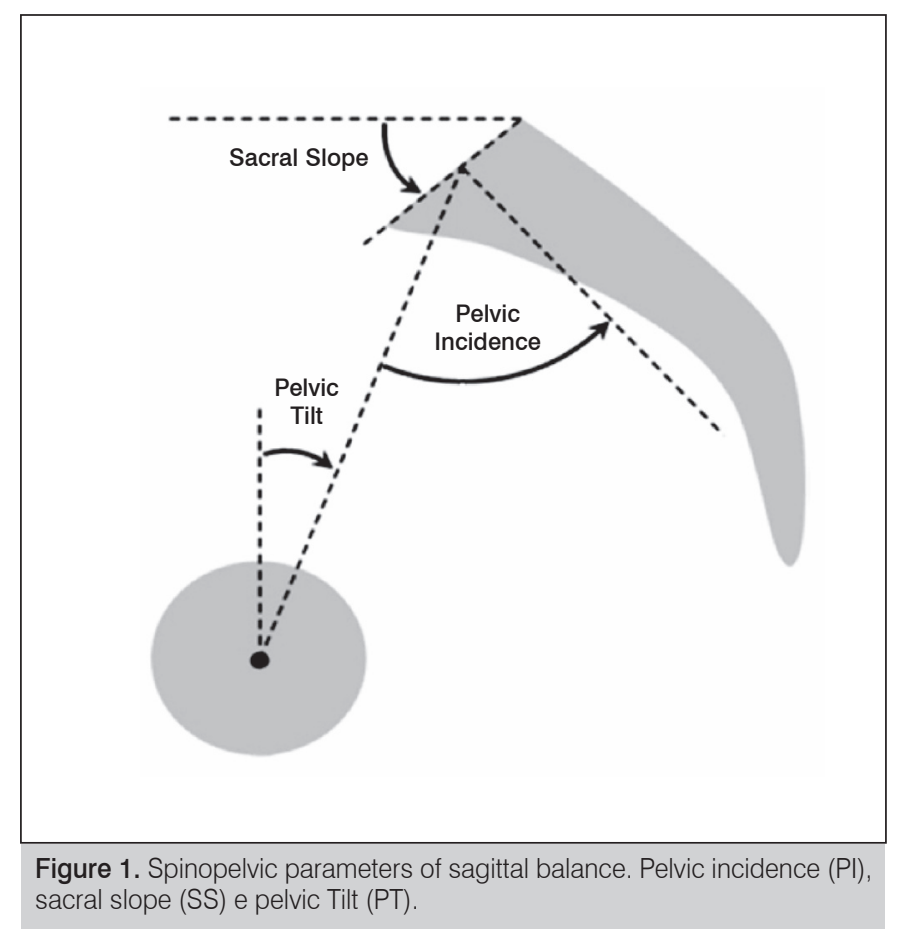

\section{RESULTS}

The sample analysis resulted in 111 records, of which 64 were excluded due to inadequate radiographs. All data was submitted to Kolgomorov-Smirnov normality test.

The sample comprised 47 patients, of which 13 men (27.7\%) and 34 women (72.3\%) were found. The mean age was 15.19 years old (range 1-24 years old; $95 \% \mathrm{Cl} 15.37$ [14.79 to 16.87]) with a median of 16 years old. (Table 1)

We found 14 patients (29.8\%) with neuromuscular scoliosis and 33 patients (70.2\%) with idiopathic scoliosis. (Table 1)

Calculated overall Pelvic Incidence resulted on average 55.02 degrees (range 20-91 degrees, 95\% Cl 55.28 [50.56 to 59.49]) and a median of 53.50. The calculated overall Sacral Slope resulted on average 44.45 degrees (range 18-71 degrees, 95\% Cl 44.45 [40.51 to 48.40]) and a median of 44.00. Calculated overall Pelvic Tilt resulted on average 10.57 degrees (range 2-32 degrees, $95 \% \mathrm{Cl} 10.10$ [range 8.46 to 12.67]) and a median of 10.00. Calculated overall Lumbar Lordosis resulted on average 45.25 degrees ( 10 to 73 degrees, $95 \% \mathrm{Cl} 45.58$ [range 40.09 to 50.41]) and a median of 46.00 . The calculated overall Thoracic kyphosis resulted on average 36.11 degrees (range 2-93 degrees, 95\% Cl 35.07 [29.80 to 42.43]) and a median of 31.50 .

\section{In the analysis by etiology, we found:}

For Idiopathic Scoliosis: The calculated overall Pelvic Incidence resulted on average 55.55 degrees (range 24-91 degrees, $95 \% \mathrm{Cl} 55.47$ [50.51 to 60.58]) and a median of 53.00. The calculated overall Sacral Slope resulted on average 45.35 degrees (range 21-69 degrees, 95\% Cl 45.39 [40.89 to 49.82]) and a median of 45.00. Calculated overall Pelvic Tilt resulted on average 10.19 degrees (range 2-24 degrees, $95 \% \mathrm{Cl} 9.96$ [7.90 to 12.49]) and a median of 10.00. The calculated overall Lumbar lordosis resulted on average 43.48 degrees (range 10 to 70 degrees, $95 \% \mathrm{Cl}$ 43.79 [37.60 to 49.37]) and a median of 46.00. The calculated overall Thoracic Kyphosis resulted on average 32.10 degrees (range 2-62 degrees, 95\% Cl 32.05 [26, 26-37-94]) and a median of 30.00 .

For neuromuscular scoliosis: the calculated overall Pelvic Incidence resulted on average 53.77 degrees (range 43 to 32 degrees, $95 \% \mathrm{Cl} 54.41$ [43.32 to 64.22]) and a median of 55.00. The calculated overall Sacral Slope resulted on average 42.31 degrees (range 18-71 degrees, 95\% Cl 42.06 [33.25 to 51.37]) and a median of 43.00. Calculated overall Pelvic Tilt resulted oon averagen average of 11.46 degrees (range 2-32 degrees, $95 \% \mathrm{Cl} 10.10$ [6.31 to 16.61]) and a median of 10.00. The calculated overall Lumbar Lordosis resulted

\begin{tabular}{c|c|c|c|c}
\multicolumn{1}{c|}{ Table 1. Sample characterization. } \\
\hline & Mean & Median & Cl 95\% & $\begin{array}{c}\text { Proportion } \\
\text { (number) }\end{array}$ \\
\hline Age (years old) & 15,19 & 16,00 & $15,37(14,79-16,87)$ & \\
\hline Idiopathic Scoliosis & - & - & - & $70,2 \%(33)$ \\
\hline Neuromuscular Scoliosis & - & - & - & $29,8 \%(14)$ \\
\hline Feminine & - & - & - & $72,3 \%(34)$ \\
\hline
\end{tabular}


on average 49.46 degrees (range 15 to 73 degrees, $95 \% \mathrm{Cl}$ 50.07 [37.99 to 60.93]) and a median of 53.00. The calculated overall Thoracic Kyphosis resulted on average 45.69 degrees (range 7-93 degrees, 95\% Cl 45.21 [28.91 to 62.48]) and a median of 40.00 .

There was no statistically significant difference between the two groups in pelvic incidence, sacral slope, pelvic tilt, lumbar lordosis and thoracic kyphosis according to the Mann-Whitney test $(p=0.057)$ and to $T$ test, resulting in equity among all studied parameters $(p>0.005)$.

The association between Pelvic Incidence and Lumbar Lordosis was low in the general population, according to Pearson's correlation test $(R=0.121)$. (Figure 1$)$

The association between Pelvic Incidence and Lumbar Lordosis was lower among patients with neuromuscular scoliosis, according to Pearson's correlation test $(R=0.274)$ and even lower among patients with idiopathic scoliosis $(R=0.074)$. (Figure 2)

\section{DISCUSSION}

The study of sagittal balance is a current topic of discussion for the treatment of pathologies of the spine. Neuromuscular scoliosis is among the least studied populations, among those in need of treatment of spinal deformities.

The sample comprised 47 patients with a higher proportion of women compared to men, with an average age of 15.19 years old. We also found a higher proportion of patients with idiopathic scoliosis than patients with neuromuscular scoliosis (29.8\%), but the groups were statistically comparable.

In the comparative analysis, very similar data to the parameters described by Li et al. ${ }^{2}$ for the general population were found. The measures also varied little between the two populations studied. These findings lead us to believe that possibly the generation of deformities in neuromuscular diseases do not affect significantly the spinopelvic parameters. Therefore, spine deformities in neuromuscular scoliosis are accompanied by important pelvic deviations in the coronal plane, without significantly affecting the sagittal plane.

The constancy of spinopelvic parameters in the population is of great importance because it is used as a guide in planning the surgical correction of deformities in the sagital plane., ${ }^{2,5}$

In order to increase sample validation, association between Pelvic Incidence and Lumbar Lordosis was done, which has already been described in the literature in other populations of patients with idiopathic scoliosis. This analysis had never been done in a Brazilian population of patients with idiopathic scoliosis nor in neuromuscular scoliosis patients in other countries.

In this study, a low association between Pelvic Incidence and Lumbar Lordosis in the population of patients with neuromuscular scoliosis and an even lower association was found in these parameters in the population of patients with idiopathic scoliosis. This result is contrary to the data reported in the literature that have reported a greater association between the two parameters in idiopathic scoliosis. ${ }^{1,8}$ Although low, the association was statistically significant, being possible its increase with the increase of the sample.

Given the large number of excluded patients, we obtained a small sample, which may have contributed to the low statistical

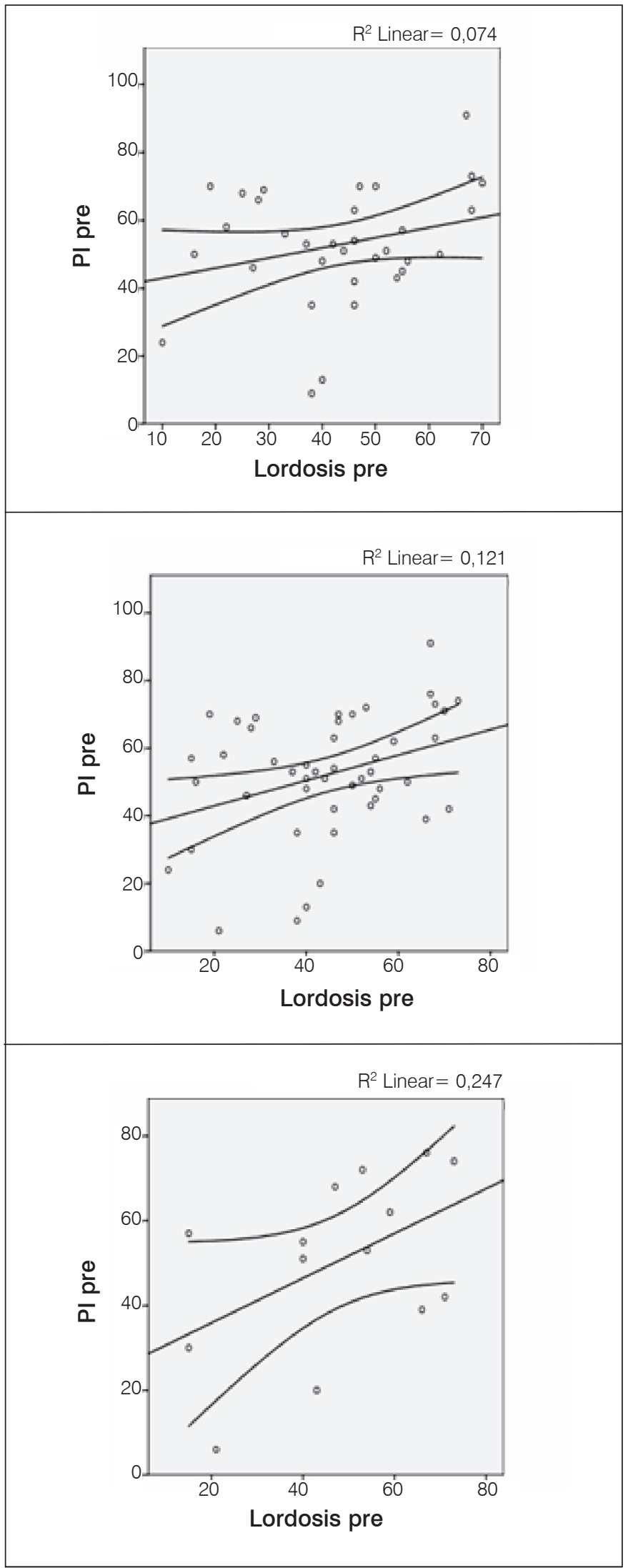

Figure 2. Correlation and dispersion charts between pelvic incidence and lumbar lordosis on the overall study sample (top), among patients with idiopathic scoliosis (middle) and among patients with neuromuscular scoliosis (bottom).Charts show a poor association between data studied. 
power of the study, reflecting a non-ideal scenario for obtaining and archiving of data found in the national scenario.

This study is among the first to address the epidemiology of sagittal balance in a Brazilian population. It brings information that forms the basis for understanding the influence and epidemiology of spinopelvic parameters in this specific population and establishes a bridge for future studies of treatment of these patients.

\section{CONCLUSION}

The spinopelvic parameters measured in patients with idiopathic and neuromuscular scoliosis in Brazilian population are similar to those described in the international literature and do not significantly vary from each other, yet the association between Lumbar Lordosis and Pelvic Incidence cannot be confirmed in this population sample.

\section{REFERENCES}

1. Mac-Thiong JM, Labelle H, Roussouly P. Pediatric sagittal alignment. Eur Spine J. 2011;20(Suppl 5):586-90.

2. Li WS, Li G, Chen ZQ, Wood KB. Sagittal plane analysis of the spine and pelvis in adult idiopathic scoliosis. Chin Med J (Engl). 2010;123(21):2978-82.

3. Kuntz C 4th, Shaffrey Cl, Ondra SL, Durrani AA, Mummaneni PV, Levin LS, et al. Spinal deformity: a new classification derived from neutral upright spinal alignment measurements in asymptomatic juvenile, adolescent, adult, and geriatric individuals. Neurosurgery. 2008;63(Suppl 3):25-39.

4. Voutsinas SA, MacEwen GD. Sagittal profiles of the spine. Clin Orthop Relat Res. 1986;(210):235-42.

5. Mac-Thiong JM, Labelle H, Berthonnaud E, Betz RR, Roussouly P. Sagittal spinopelvic balance in normal children and adolescents. Eur Spine J. 2007;16(2):227-34.
6. Glassman SD, Bridwell K, Dimar JR, Horton W, Berven S, Schwab F. The impact of positive sagittal balance in adult spinal deformity. Spine (Phila Pa 1976). 2005;30(18):2024-9.

7. Mac-Thiong JM, Transfeldt EE, Mehbod AA, Perra JH, Denis F, Garvey TA, et al. Can c7 plumbline and gravity line predict health related quality of life in adult scoliosis? Spine (Phila Pa 1976). 2009;34(15):E519-27.

8. Tanguay F, Mac-Thiong JM, de Guise JA, Labelle H. Relation between the sagittal pelvic and lumbar spine geometries following surgical correction of adolescent idiopathic scoliosis. Eur Spine J. 2007;16(4):531-6.

9. Cobb JR. Outline for the study of scoliosis. Instr Course Lect. 1948;5:261-75.

10. Kuklo TR, Potter BK, Schroeder TM, O'Brien MF. Comparison of manual and digital measurements in adolescent idiopathic scoliosis. Spine(Phila Pa 1976).2006;31(11):1240-6. 